\title{
Research on Convolution Neural Network in Iris Recognition Technology
}

\author{
Wenqiang Zhang \\ School of Computer Science and Engineering \\ Xi'an Technological University \\ Xi'an, 710032, China \\ e-mail: 757712672@qq.com \\ Changyuan Wang \\ School of Computer Science and Engineering \\ Xi'an Technological University \\ Xi'an, 710032, China
}

\author{
Hongbo Jia \\ Air Force Aviation Medical Research Institute \\ Beijing, China
}

Pengxiang Xue

School of Computer Science and Engineering

Xi'an Technological University

Xi'an, China

\begin{abstract}
The iris recognition is mainly aimed at iris matching, and everyone has a different iris. In this paper, design an efficient Convolutional Neural Network for iris recognition. First need to preprocess the iris image and the iris region of the eye is extracted. And then, divide the iris area into eight rectangular regions, those sub-regions are put into the designed model, which can extract the iris abstract features and identify the iris. Finally, train the model by collecting large amounts of data and that the correctness of the model can reach about $99.0 \%$, which can meet the requirement of iris recognition effectively.
\end{abstract}

Keywords-Iris; Convolution Neural Network; Model; Abstract Features

\section{INTRODUCTION}

In recent years, bio-identification technology has been developed in the world, including speech recognition, iris recognition and facial recognition[5] and so on, which are different from the traditional authentication methods. Among these emerging technologies, iris recognition is one of the most challenging research. The iris recognition has many advantages: (1) Irisrecognitionhas isolated and protected ability; (2) iris structure can not be modified; (3) the light has its own physiological response, which can exclude false iris.

There are two typical algorithms in traditional iris recognition: One is proposed by Dr. John G Daugmanin Cambridge University and the other by Dr. Richard $\mathrm{P}$ Wildesin MIT Artificial Intelligence Laboratory. Dr. Daugman uses the Gabor filter to make a rough quantization and code the iris texture[6], however it is sensitive to the rotation and drift of the iris image; and the Wildes system is based on image registration techniques and it calculation is very larger. In this paper proposes a new iris recognition method. It can extract the iris region of the eye image and gets the divided eight rectangular regions, and thenthe eight rectangular regions are input the model, finally, the iris image is identified. By using the method of this paper the iris image can be effectively identified and classified, and the accuracy can reach $99.0 \%$, and the speed of the model is very fast.

\section{PRETREATMENT OF IRIS IMAGE}

The purpose of the preprocessing of the iris image is to separate the iris area from the eye image, and divide the iris area into eight rectangular regions. Finally, we use image enhancement to improve the area quality.

\section{A. Iris Area Extraction}

As shown in Figure 1, the iris areainclude inside and outside rounded edge of the circular, and two circle of the center can be approximated as the same point, where the internal circle is a solid circle of the pupil. So the pupil circle can be detected, and then through the center of the pupil to expand the radius, and to find the outer edge of the iris contour, finally extract the iris area.

Firstly, smooth the eye image and transformed into a binary image by thresholding, then, the pupil circle is identified by using the Hough transform. According to the proportion of the eye image and the center coordinates of the pupil, the radius of the extended circle is the appropriate size to obtain the outer edge of the iris image. The portion between the two rounded edges can be as the iris area,the extractionresult is shown in Figure 2.

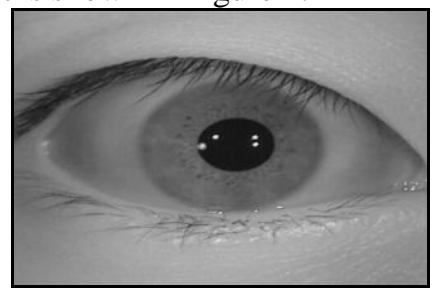

Figure 1. Image of human eye

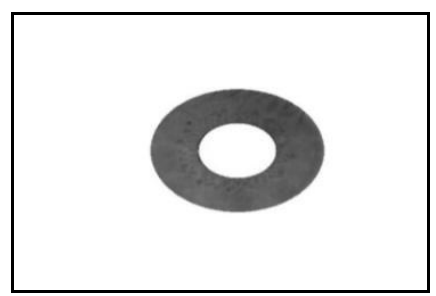

Figure 2. Feature extraction result of iris image 


\section{B. Rectangular division of iris area}

In the iris image to divide eight rectangular regions of the same size and as the input of the model. Each rectangular region contains iris shape and texture information, which can provide important value for iris recognition. The rectangular region division result is shown in Figure3.

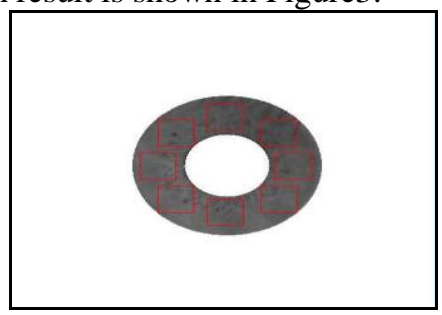

Figure 3. Division block of iris area

\section{Iris image enhancement}

The rectangular texture of the iris image is not large enough, Preprocessing with homomorphic filtering[9], and use the appropriate filter characteristic function, so that the final input image has a strong contrast, and the enhanced rectangular region of the iris image is shown in Figure4.

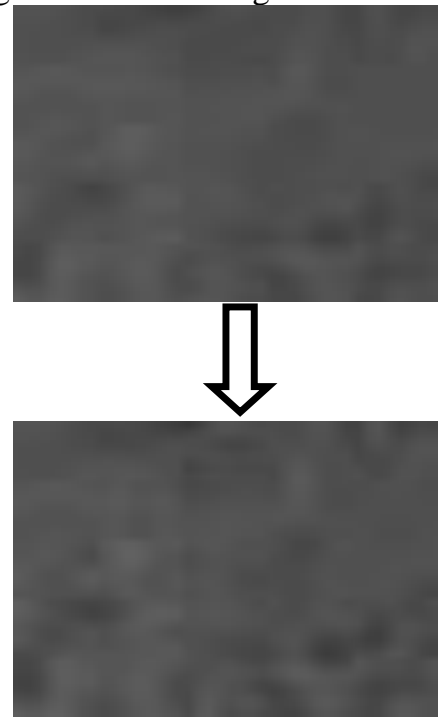

Figure 4. Iris image enhancement

\section{IRIS RECOGNITION BASED ON CONVOLUTION NEURAL NETWORK}

\section{A. Iris Recognition Basic Process}

In this paper, according to the iris recognition model, the flow chart of iris recognition is shown in Figure 5

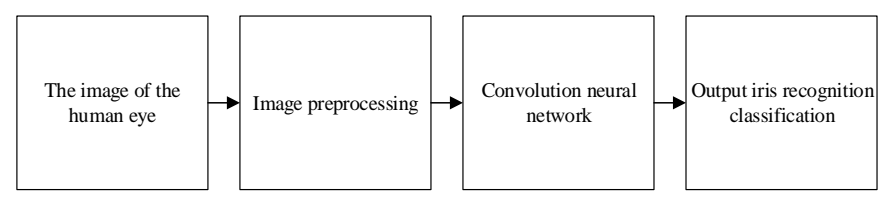

Figure 5. Iris identification schematic flow chart

\section{B. Characteristics of Convolution Neural Network}

Convolution neural network is a multi-layer perceptron. The main reason why convolution neural network is widely used in image recognition technology is that it adopts two mechanisms: local connection and weight sharing. Local connection refers to the neuron of each layer of network structure only with a layer of its designated size of the nerve element connection, through the convolution block to give each neuron weight, which extracted the characteristics of the target image. Weight sharing means that the neurons corresponding to the same feature map share the same weight, which reduces the number of training parameters required.

The main operations of convolution neural networks contain: Convolution and pooling. The volume network is used as input to the output feature in the upper layer network, and then convolution operation is carried out with the convolution kernel. Finally, the input function of the next layer is obtained by an activation function. The mathematical formula is as follows:

$$
x_{j}^{k}=f\left(\sum_{i \in M_{j}} x_{i}^{k-1} * w_{i j}^{k}+b_{j}^{k}\right)
$$

where $x_{j}^{k}$ represents the $j$-th feature of the $k$-th layer, ${ }^{M_{j}}$ is the set of selected features, $x_{i}^{k-1}$ is the $i$-thfeature image of the $(k-1)$-th layer, ${ }^{k}{ }_{i j}^{k}$ is the weight of between the ${ }^{j}$-th features of the $k$-th layer and the $j_{\text {-th features of }}$ the $(k-1)$-th the layer, $b_{j}^{k}$ represents the parallax corresponding to the $j$-th feature of the $k$-th layer, and $f$ corresponds to the activation function. The activation function used in this paper is the ReLU function, which is defined as:

$$
f(x)=\max (0, x)
$$

where $x$ represents the input of the neuron.

With the pool layer of the neural network, the feature of the image can be reduced, which can reduce the number of nodes in the network and improve the speed of the neural network. The convolution layer and the pool layer of the neural network can extract the characteristics of the iris image automatically, and the iris image is identified and classified at the end of the neural network.The full connection layer of this paper is two layers, and the classifier is Softmax classifier. The input of the full connection layer is the output of the previous process feature vector extraction. In order to prevent the over-fitting problem of the model, we need to limit the weight of the whole connection layer. The method used in this paper is $\mathrm{L} 2$ regularization method, the formula is as follows:

$$
\mathrm{R}(w)=\|w\|_{2}^{2}=\sum_{i}\left|w_{i}^{2}\right|
$$

where $w$ is the weight of the fully connected layer.

The Softmax classifier is added to the output layer of the neural network, and the prediction results of the neural 
network are probabilized. Then, the final classification result of the iris is determined according to the predicted probability. The output after Softmax classification is:

$$
\operatorname{soft} \max \left(y_{i}\right)=y_{i}^{\prime}=e^{y_{i}} / \sum_{j=1}^{n} e^{y_{j}}
$$

where $y_{i}$ is the original $i$-th output of the neural network.

According to the final output of the model, this paper uses the cross entropy loss function to calculate the loss of the prediction results. In the process of model training, through the parameters of the model continue to train updates, the forecast loss is reduced, and ultimately achieve the desired results. In this paper, the learning rate of the model is set by using the exponential decay method, so that the model can quickly reach a better solution. At the same time, in order to prevent the over-fitting problem of the model, this paper chooses the forecast value of the model. This paper uses the Dropout method provided in Tensorflow. Where the model is validated and the correct rate of real-time training is obtained. Finally, the model is evaluated by the test data to verify the accuracy of the model.

\section{Convolution neural network structure}

The convolution neural network designed in this paper is used for iris recognition. The structure of the network is shown inFigure 6. It can be seen that the neural network consists of three convolution layers, three pool layers, two full connection layers and one Softmax layer.

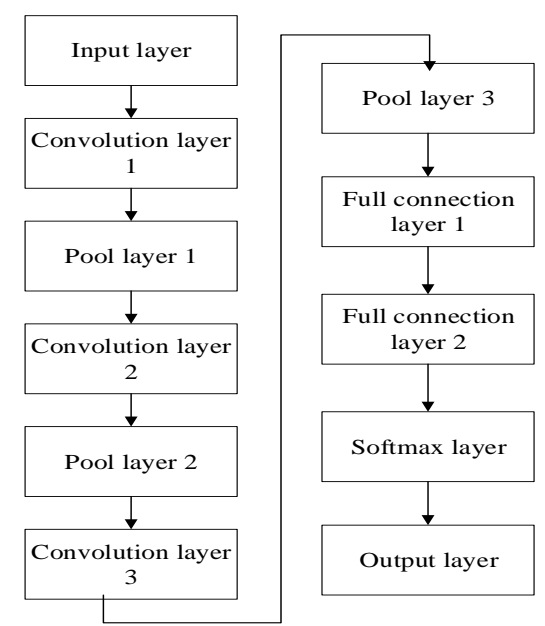

Figure 6. Structure of convolution neural network

\section{EXPERIMENTAL RESULTS AND ANALYSIS}

In order to verify the superiority of the model, the CASIA iris database is used as the data set. The method is implemented in Python language with Tensorflow platform, and the PC memory is $8 \mathrm{G}$.

\section{A. the processing of the input data set}

The iris image of the data set is pretreated and put into the convolution neural network model to train the model. At the end of the model training, some of the human eye images in the CASIA iris database are used to validate the model.
The data set used in the test model is the iris image in the CASIA iris database.

\section{B. Experimental process and result analysis}

The overall process of this experiment is as Figure 7:

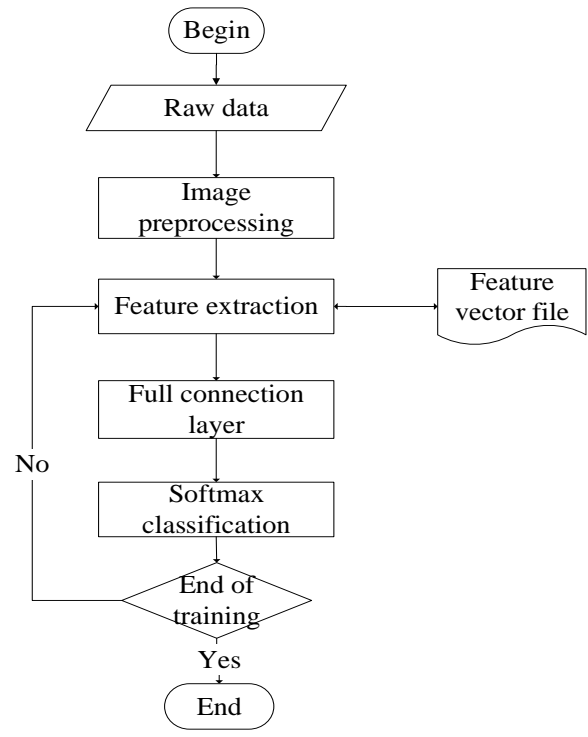

Figure 7. Experimental flow chart

In this paper, the batch sizeis 50, The basic parameters set at the beginning of the training are shown in table I:

TABLE I. The Initial Parameters of The Model

\begin{tabular}{ccccc}
\hline $\begin{array}{c}\text { Verify the } \\
\text { percentage } \\
\text { of data }\end{array}$ & $\begin{array}{c}\text { Percentage } \\
\text { of test } \\
\text { data }\end{array}$ & $\begin{array}{c}\text { Learning } \\
\text { rate }\end{array}$ & $\begin{array}{c}\text { Number of } \\
\text { training } \\
\text { rounds }\end{array}$ & $\begin{array}{c}\text { Batch } \\
\text { size }\end{array}$ \\
\hline 10 & 10 & 0.01 & 2000 & 50 \\
\hline
\end{tabular}

The model training results are shown in Figure 8:

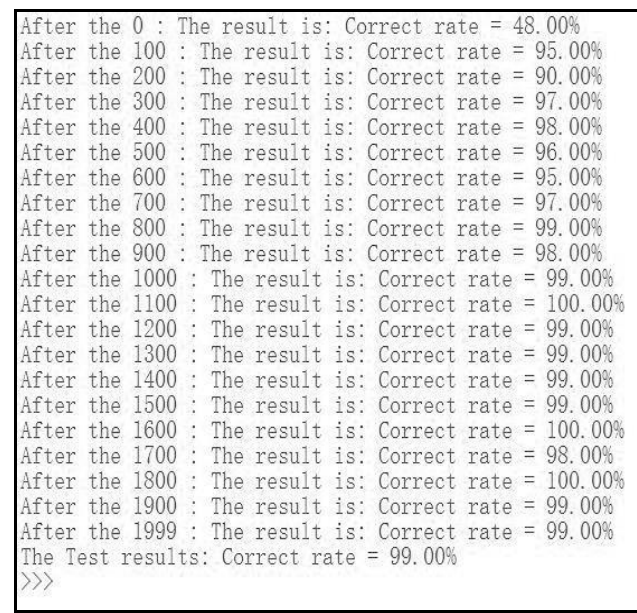

Figure 8. Model training results

As shown in Figure 8, the initial correct rate of the model is $48 \%$ before the model starts training. After that, the verification model is validated against the current model for 
100 rounds of training and the verification result is output. After 100 rounds of training, the correct rate of the model immediately increased to $95 \%$, we can see that learning speed of the model is very fast. The accuracy of the final model is basically maintained at about $99 \%$, which can meet the basic application requirements of iris recognition.

With the continuous training of the model, the loss function of the model is decreasing and the prediction accuracy of the model is gradually increasing. The Figure 9 and Figure 10shows the change trend of the model's loss function and prediction accuracy.

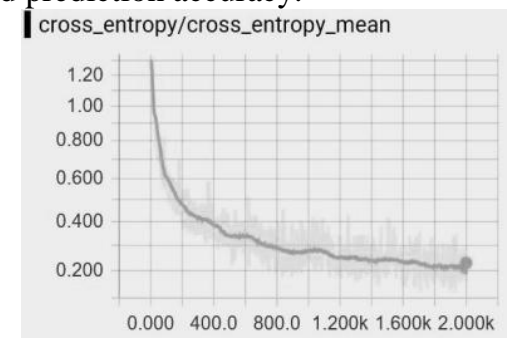

Figure 9. Loss function change trend result

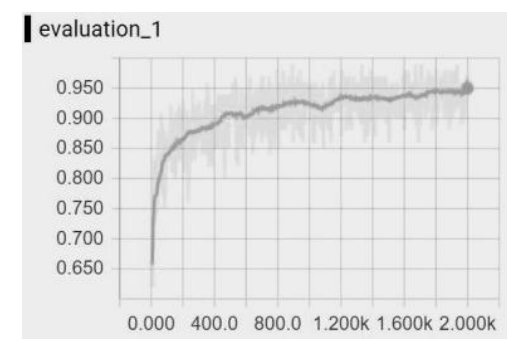

Figure 10. Predictive accuracy rate change trend result

Through the experiment, the identification accuracy of the convolution neural network modeland the traditional neural network model are compared. Meanwhile the partical results are shown in the following table II:

TABLE II. Comparison of Model Training Results

\begin{tabular}{ccc}
\hline Model method & $\begin{array}{c}\text { data set } \\
\text { Number of training } \\
\text { rounds }\end{array}$ & Accuracy \\
\hline $\begin{array}{c}\text { Literature [2] } \\
\text { method }\end{array}$ & 2000 & $93.8 \%$ \\
$\begin{array}{c}\text { Literature [3] } \\
\text { method }\end{array}$ & 2000 & $98.7 \%$ \\
The paper method & 2000 & $99.0 \%$ \\
\hline
\end{tabular}

Through the comparison of the above results, the efficiency of this method is improved obviously. Under the same training rounds, the accuracy of the model is improved obviously. The parameters in the model are also reduced greatly compared with the traditional neural network.

\section{CONCLUSION}

In this paper, used the preprocessing of data set, extract the feature of the iris area and divide it into eight rectangular sub-regions, which can overcome the occlusion influence effectively. With the enhancement processing of the image, it can provide effective help for recognition. During the training of the convolution neural network model, and to extract the iris image data set. The neural network uses only two whole connection layer, which can decrease the number of parameters in the network, and further improve the training speed of greatly.Meanwhile, the regularization method and the Dropout method is adopted in the training process, which reduces the over-fitting problem of the model and makes it more effective in identifying the iris image.

However, this paper method still exist some shortcomings, In the model, the data set of different experimental object iris image is read randomto improve the randomness, but it is difficult to output current recognition results belong to which category. To solve such problems, it still need further study in the future.

\section{ACKNOWLEDGMENT}

This paper is supported by the local special program of the Shaanxi Provincial Department of Education (project number: 16JF012).

\section{REFERENCES}

[1] Fuqing Pu, Image Recognition and Classification Based on Artificial Neural Network,Chengdu: Chengdu University of Technology, 2010.

[2] Yunhong Wang, yong Zhu, Tieniu Tan,Recognition based on iris recognition,1rd ed., Vol.28. Journal of Automation,2002, pp. 1-10.

[3] Weiqi Yuan, Qi Feng, LiKe, An Iris Recognition Method for Extracting Texture Orientation Using 2D - Gabor Filter, 8rd ed., Vol.26. Computer Application Research,2009, pp.3166-3168.

[4] Zhihong Zhao, Shaopu Yang, Zengqiang Ma, Research on License Plate Character Recognition Based on Convolution Neural Network LeNet-5,3rd ed.,Vol.22.Journal of System Simulation, 2010, pp.638641.

[5] Richard P W,Jane C A,Gilbert L G,etal.A machine vision system for iris recognition, 1rd ed., Vol.9.Machine vision and Application, 1996, pp.1-8.

[6] J.G Daugman.Biomedical personal identification system based on iris analysis.U S,Patent,5291560, 1994.

[7] Hui Li, Bo Shi, Face Recognition Algorithm Based on Convolution Neural Network,3rd ed., Vol.16.Software Guide,2017, pp.26-29.

[8] WeiliangZeng,ZhixianLin, Yongsa Chen, Research on Image Recognition of Fruit and Vegetable in Intelligent Refrigerator Based on Convolution Neural Network,8rd ed., Vol.36.Microcomputer and Application, 2017, pp.56-59.

[9] Xinming Zhang, Lansun Shen, Wavelet - based Homomorphic Filters for Image Contrast Enhancement,4rd ed., Vol.29.Journal of Electronics, 2001, pp.531-533

[10] Jing Wang, Research on License Plate Character Recognition Technology Based on Deep Learning Neural Network,3rd ed., Vol.30.Industrial Control Computer, 2017, pp.51-52.

[11] Yumin Wang, NaijieGu, Xiaoci Zhang, Convolution Neural Network Parallel Algorithm for Multi - GPU Environment,3rd ed. Vol.38.Small microcomputer system, 2017, pp.536-539.

[12] JiaY,ShelhamerE,DonahueJ,etal.Caffe:Convolutional architecture for fast feature embedding.Proceedings of the 22nd ACM International Conference on Multimedia,2014, pp.675-678.

[13] Erlei Hu, Rui Feng, Image Retrieval System Based on Depth Learning,30rd ed., Vol.26.Computer system application,2017, pp.819.

[14] FARIA F A,dos SANTOS J A,ROCHA A,etal.Automatic classifier fusion for produce recognition .25th SIBGRAPI on Graphics,Patterns and Images, OuroPreto,Brazil,IEEE,2012, pp.20-25.

BENGIO Y.Learning deep architectures for AI.Foundations and Trends in Machine Learning, 1rd ed., Vol.2.2009,pp.1-127. 